\title{
Avaliação da atividade fotoprotetora do extrato aquoso de Rhaphiodon echinus (Nees \& Mart.) Schauer
}

Evaluation of the photoprotective activity of the aqueous extract of Rhaphiodon enchinus (Nees \& Mart.) Schauer

\author{
M. A. C. Medeiros ${ }^{1 *}$; B. Santos ${ }^{1}$; F. M. C. Marques ${ }^{1}$; M. F. M. S. Leite ${ }^{1}$; M. M. \\ Simões $^{1}$; R. M. Anjos ${ }^{1}$; L. Brito Júnior ${ }^{1}$; G. L. A. Maia ${ }^{2}$; M. A. S. G. Alves ${ }^{1}$; A. P. \\ Sousa $^{3}$; A. A. Oliveira Filho ${ }^{1}$ \\ ${ }^{1}$ Universidade Federal de Campina Grande, Centro de Saúde e Tecnologia Rural, 58708-110, Patos-PB, Brasil \\ ${ }^{2}$ Universidade Federal do Vale do São Francisco, 56304-205, Petrolina-PE, Brasil \\ ${ }^{3}$ Departamento de Fisiologia e Patologia, Centro de Ciências da Saúde, Universidade Federal da Paraíba, 58033-455, \\ João Pessoa-PB, Brasil
}

*mauricioandre64@gmail.com

(Recebido em 01 de fevereiro de 2021; aceito em 16 de abril de 2021)

\begin{abstract}
A pele é o maior órgão do corpo e possui diversas funções no organismo: proteção, nutrição, pigmentação e absorção. A exposição frequente aos raios solares causa sérios problemas dermatológicos, que são estimulados pela incidência da radiação ultravioleta. Umas das alternativas para inibir os efeitos dos raios ultravioleta é a utilização de protetor solar. Na estruturação de alternativas que possam produzir efeito fotoprotetor, a utilização de produtos derivados de plantas ganha resultados positivos. O metabolismo secundário de inúmeras plantas produz compostos orgânicos com efeito sinérgico na fotoproteção. Os flavonóides são metabólitos secundários que integram os compostos fenólicos. A espécie Rhaphiodon echinus, conhecida como "bentônica" apresenta flavonoides em sua composição bioquímica. O presente estudo objetivou avaliar o potencial fotoprotetor, in vitro, do extrato aquoso de $R$. echinus. Foi utilizado o extrato aquoso da planta em diferentes concentrações 50,100,500 e $1000 \mu \mathrm{g} / \mathrm{mL}$ e realizado varreduras em espectrofotômetro no comprimento de onda de 290 a $320 \mathrm{~nm}$ com intervalos de $5 \mathrm{~nm}$ a cada 5 minutos. Os resultados foram calculados segundo à equação de Mansur para determinar o fator de proteção solar. O produto apresentou fotoproteção em todas as concentrações avaliadas $>6_{\mathrm{FPS}}$, as concentrações de 500 a 1000 $\mu \mathrm{g} / \mathrm{mL}$ obtiveram maior efeito fotoprotetor com $>25_{\mathrm{FPS}}$. No entanto, torna-se necessário mais estudos fitoquímicos e farmacológicos para melhor elucidar as moléculas presentes nos extratos de $R$. echinus e sua atividade biológica.
\end{abstract}

Palavras-chave: fotoproteção, Rhaphiodon echinus, extrato aquoso.

The skin is the largest organ in the body and has several functions in the body: protection, nutrition, pigmentation and absorption. Frequent exposure to sunlight causes serious dermatological problems, which are stimulated by the incidence of ultraviolet radiation. One of the alternatives to inhibit the effects of ultraviolet rays is the use of sunscreen. When structuring alternatives that can produce a photoprotective effect, the use of products derived from plants gains positive results. The secondary metabolism of numerous plants produces organic compounds with a synergistic effect on photoprotection. Flavonoids are secondary metabolites that integrate phenolic compounds. The species Rhaphiodon echinus, known as "bentônica" has flavonoids in its biochemical composition. The present study aimed to evaluate the photoprotective potential, in vitro, of the aqueous extract of $R$. echinus. The aqueous extract of the plant was used in different concentrations 50,100,500 and $1000 \mu \mathrm{g} / \mathrm{mL}$ and spectrophotometer scans were performed at a wavelength of 290 to $320 \mathrm{~nm}$ with $5 \mathrm{~nm}$ intervals every 5 minutes. The results were calculated according to Mansur's equation to determine the sun protection factor. The product showed photoprotection at all concentrations evaluated $>6$ SPF, concentrations from 500 to $1000 \mu \mathrm{g} / \mathrm{mL}$ had a greater photoprotective effect with $>25$ SPF. However, more phytochemical and pharmacological studies are needed to better elucidate the molecules present in R. echinus extracts and their biological activity.

Keywords: photoprotection, Rhaphiodon echinus, aqueous extract.

\section{INTRODUÇÃO}

A pele é o maior órgão do corpo humano e apresenta diversas funções como proteção, nutrição, termorregulação, pigmentação e absorção. A produção de vitamina $\mathrm{D}$ é outra função importante 
que depende da incidência da radiação solar sob a pele, desencadeia reações que levam à produção dessa vitamina assegurando absorção no intestino do: cálcio, fósforo, garantindo o crescimento e a reparação dos ossos, o funcionamento celular e neuromuscular $[1,2]$.

A radiação ultravioleta, proveniente da incidência dos raios solares, alcança a superfície da terra, podendo ser influenciada pela variação latitudinal, período sazonal e nível de altitude em que se encontra, destacando-se as radiações UV-A (intervalo de $400-320 \mathrm{~nm}$ ) e UV-B (intervalo de 320 - $280 \mathrm{~nm}$ ), que pode causar problemas a saúde das pessoas, a depender do período de exposição [3]. A exposição frequente à radiação solar pode desencadear diversos problemas como eritema ou queimadura solar, pigmentação solar, fotoenvelhecimento e câncer de pele [4, 5].

De acordo com Sonda (2011) [6] características genéticas e a exposição à radiação solar são fatores importantes para o desenvolvimento do melanoma, um tipo de câncer de pele que apresenta letalidade elevada. Nesse contexto, o uso de fotoprotetores é fundamental para a prevenção de todas as neoplasias de pele.

As plantas possuem muitas propriedades bioquímicas com diversos efeitos benéficos para a saúde humana. Muitos extratos e óleos de plantas têm sido utilizados em produtos cosméticos como filtros solares, devido à ação fotoprotetora. Dessa forma, muitas plantas apresentam potencial importante para inibir os efeitos danosos dos raios solares com menor custo por ser natural e sustentável [7, 8].

O metabolismo secundário das plantas sintetiza vários compostos orgânicos fundamentais para sobrevivência das plantas e que fornecem substâncias valiosas a saúde humana. Os flavonoides, por exemplo, possuem ação contra os raios ultravioletas e estão envolvidos na determinação do tom de cor do órgão reprodutor de angiospermas [9]. Os flavonoides, em extratos derivados de plantas, apresentam um bom desempenho contra os efeitos da radiação ultravioleta, levando em consideração, também, sua capacidade antioxidante, atuando no mecanismo de inibição e propagação de radicais livres [10].

A espécie Rhaphiodon echinus, que pode ser encontrada no bioma Caatinga, é endêmica do Brasil, sendo atualmente um gênero monotípico, pertencendo a família Lamiaceae [11]. Conhecida popularmente como "betônica", apresenta indicação para tratamento de infecção do trato urinário e dismenorréia. Essa planta apresenta diversos metabólitos secundários como taninos, saponinas e flavonoides [12]. No entanto, há escassez de estudos sobre isolamento e purificação de moléculas com Rhaphiodon echinus deve ser considerada. Contudo, o presente estudo buscou reforçar a relevância sobre o conhecimento da família Lamiaceae e da planta.

Baseado nas informações expressadas à utilização de produtos naturais e à fotoproteção, esse trabalho tem como objetivo avaliar, in vitro, o potencial fotoprotetor do extrato aquoso da espécie Rhaphiodon echinus.

\section{MATERIAL E MÉTODOS}

\subsection{Extrato Vegetal}

Para o desenvolvimento do estudo in vitro, utilizou-se o extrato aquoso que foi ofertado pela equipe da Prof $^{a}$. Dr ${ }^{a}$. Gabriela Lemos de Azevedo Maia da Universidade Federal do Vale do São Francisco.

\subsection{Avaliação do Fator de Proteção Solar do Extrato Aquoso de Rhaphiodon echinus}

A análise espectrofotométrica de absorção utilizando o extrato aquoso da espécie Rhaphiodon echinus, realizou-se no espectro da radiação ultravioleta como proposto por Mansur et al. (1986) [13]. Desse modo foi efetuado varreduras de 290 a $320 \mathrm{~nm}$, acrescentando a isso, intervalos de $5 \mathrm{~nm}$ com duração de 5 minutos. Ao final de cada intervalo foi realizada a mensuração das absorbâncias.

Na obtenção da leitura, utilizou-se o espectrofotômetro digital (Biospectro®) com cubeta de quartzo de $1 \mathrm{~cm}$. Realizada a mensuração das absorbâncias, os dados extraídos foram submetidos a equação de Mansur et al. (1986) [13] para verificar o Fator de Proteção Solar (FPS) in vitro. Esse 
método coloca em lista o efeito eritomatogênico e a intensidade da radiação (EE x I) que foram medidos por Sayre et al. (1979) [14]. Esses são demonstrados na Tabela 1, logo abaixo:

Tabela 1: Relação efeito eritemogênico (EE) versus intensidade da radiação (I) conforme o comprimento

\begin{tabular}{c|c}
\multicolumn{2}{c}{ de onda $(\lambda)}$. \\
\hline$\lambda / \mathbf{n m}$ & EE x I \\
\hline 290 & 0,0150 \\
295 & 0,0817 \\
300 & 0,2874 \\
305 & 0,3278 \\
310 & 0,1864 \\
315 & 0,0839 \\
320 & 0,0180
\end{tabular}

Fonte: Sayre et al. (1979) [14]

Sendo que a fórmula de Mansur et al. (1986) [13] é também composta pela leitura espectrofotométrica da absorbância da amostra e fator de correção $(=10)$. Essa equação pode ser observada, a seguir:

$$
\text { FPS espectrofotométrico }=\text { FC. } \sum_{290}^{320} \mathrm{EE}(\lambda) . \mathrm{I}(\lambda) . \text { Abs }(\lambda)
$$

Na qual: FPS = fator de proteção solar; FC = fator de correção, calculado de acordo com dois filtros solares de FPS conhecidos e testados em seres humanos de tal forma que um creme contendo $8 \%$ de homossalato resultasse no $\operatorname{FPS} 4 ; \operatorname{EE}(\lambda)=$ efeito eritemogênico da radiação de comprimento de onda; $\mathrm{I}(\lambda)=\mathrm{a}$ intensidade da luz solar no comprimento de onda e Abs $(\lambda)=$ a absorbância da amostra no comprimento de onda.

Todo o experimento foi realizado em triplicata, como branco foi utilizado água destilada.

\section{RESULTADOS E DISCUSSÃO}

Estudos fitoquímicos realizados por Pio et al. (2019) [12] mostram que a espécie possui diversos metabólitos secundários entre eles: taninos, saponinas e flavonoides. Com a realização dessa pesquisa, como mostra a Tabela 2, o extrato aquoso de Rhaphiodon echinus apresentou valores consideráveis de fator de proteção.

Tabela 2: FPS do extrato aquoso de Rhaphiodon echinus em diferentes concentrações.

\begin{tabular}{ccccc}
\hline Concentrações $(\boldsymbol{\mu} \mathbf{g} . \mathbf{m L}-\mathbf{1})$ & $\mathbf{5 0} \boldsymbol{\mu} \mathbf{g} / \mathbf{m L}$ & $\mathbf{1 0 0} \boldsymbol{\mu} \mathbf{g} / \mathbf{m L}$ & $\mathbf{5 0 0} \boldsymbol{\mu g} / \mathbf{m L}$ & $\mathbf{1 0 0 0} \boldsymbol{\mu g} / \mathbf{m L}$ \\
\hline FPS & 6,19 & 11,15 & 25,00 & 25,00
\end{tabular}

De acordo com a RDC $N^{\circ} 30$, de $1^{\circ}$ de junho de 2012, da Agência Nacional de Vigilância Sanitária (ANVISA) [15] que aprova o regulamento técnico MERCOSUL sobre protetores solares em cosméticos e dá outras providências, atribuindo uma regulamentação técnica aplicada aos produtos e cosméticos destinados a proteção solar da pele, o fator mínimo de proteção solar tem o valor de 6 (seis) (FPS). Com base na análise dos dados apresentados na tabela 1, observa-se resultados promissores nas concentrações do extrato aquoso, pois todas ultrapassam o valor considerado mínimo de 6 para fotoproteção. De acordo com os resultados obtidos, as concentrações de 50,100, 500, e $1000 \mu \mathrm{g} / \mathrm{mL}-1$, apresentaram valores de fotoproteção igual a $6,19,11,15,25,00$ e 25,00 (FPS), respectivamente. 
Segundo Medeiros et al. (2020) [16], seguindo a mesma metodologia, porém, utilizando o extrato etanólico de Rhaphiodon echinus para verificar o potencial fotoprotetor, in vitro, obteve-se valor de fotoproteção acima de 6 (FPS) nas concentrações de $500 \mu \mathrm{g} / \mathrm{mL}-1$ e $1000 \mu \mathrm{g} / \mathrm{mL}-1$, sendo os valores, respectivamente, 23,65 e 25,00 (FPS). Outros estudos com espécies de plantas apresentam fator de fotoproteção, como, por exemplo, o estudo de Simão et al. (2019) [17], onde a espécie Praxelis clematidea (GRISEB.) R.M. King \& H. Robinson apresentou valores consideráveis de FPS, in vitro, promovendo uma possível utilização dessa planta como fotoprotetor e motivando estudos in vivo com esta espécie.

Um dos países com enorme diversidade de espécies de plantas é o Brasil, sendo a fitoterapia uma beneficiada por essa vantagem, mas, apesar de inúmeras plantas apresentarem propriedades medicinais, faz-se necessário estudos mais aprimorados dos extratos vegetais na síntese de produtos naturais e fármacos [18].

Os flavonoides são os compostos fenólicos que tem grande potencial fotoprotetor e ação antioxidante. Plantas que sintetizam esses compostos orgânicos oferecem grande diversidade de flavonoides, porém ainda não existe uma maneira singular de extrair compostos fenólicos em sua totalidade, sendo importante pesquisar a maneira de extrair tais substâncias conforme as características da planta em que se trabalhe [8].

\section{CONCLUSÃO}

Com base nos dados obtidos nessa pesquisa, pode-se observar que o extrato aquoso da espécie Rhaphiodon echinus, demonstra uma atividade promissora como fotoprotetor in vitro. No entanto, torna-se necessário mais estudos fitoquímicos e farmacológicos in vitro e in vivo para melhor elucidar os componentes presentes nos extratos de Rhaphiodon echinus e sua atividade biológica.

\section{AGRADECIMENTOS}

Os autores agradecem a Liga Acadêmica de Fitoterapia, Bioquímica e Microbiologia CSTRUFCG.

\section{REFERÊNCIAS BIBLIOGRÁFICAS}

1. Tofetti MHFC, Oliveira VRA. Importância do uso do filtro solar na prevenção do fotoenvelhecimento e do câncer de pele. Investigação. 2006 Jan;62(1):59-60, doi:10.26843/investigacao.v6i1.183.

2. Sousa RG, Lima ADS, Lima EN. Incremento da atividade fotoprotetora e antioxidante de cosméticos contendo extratos vegetais da caatinga. Braz J Nat Sci. 2020 Mar;3(1):225-230, doi: 10.31415/bjns.v3i1.83.

3. Kirchhoff VWJH, Echer E, Leme NP, Silva AA. A variação sazonal da radiação ultravioleta solar biologicamente ativa. Rev Bras de Geofísica. 2000 Mar;18(1):63-74, doi:10.1590/S0102261X2000000100006.

4. Instituto Nacional do Câncer José Alencar Gomes da Silva (INCA). Monitoramento das ações de controle do câncer de pele. Informativo: Detecção Precoce. Boletim ano 7, n. 3, set/dez 2016. Disponível em: https://www.inca.gov.br/sites/ufu.sti.inca.local/files//media/document/informativo-deteccao-precoce-32016.pdf

5. Simis T, Simis DRC. Doenças da pele relacionadas à radiação solar. Revista da Faculdade de Ciências Médicas de Sorocaba. 2006 Dez;8(1):1-8.

6. Sonda LC. Fatores de risco para melanoma: uma revisão integrativa [monografia]. Ijuí (RS): Universidade Regional do Noroeste do Estado do Rio Grande do Sul - UNIJUÍ; 2011.

7. Violante IMP, Souza IM, Venturini CL, Ramalho AFS, Santos RAN, Ferrari M. Avaliação in vitro da atividade fotoprotetora de extratos vegetais do cerrado de Mato Grosso. Rev Bras de Farmacognosia. 2009 Abr/Jun;19(2A):452-457, doi: 10.1590/S0102-695X2009000300020.

8. Carvalho JCS, Garcia PSP, Vigno SR, Pedriali CA. Estudo do impacto da utilização de ativos vegetais em fotoprotetores. InterfacEHS. 2015 Dez;10(2):62-82.

9. Vizzotto M, Krolow AC, Weber GEB. Metabólitos secundários encontrados em plantas e sua importância. Documentos, 316. Pelotas (RS): Embrapa Clima Temperado; 2010. 
10. Rosa M, Oliveira TG, Carvalho CA, Silva FD, Carvalho LM, Nascimento PC, et al. Estudo espectrofotométrico da atividade foto-protetora de extratos aquosos de Achillea millefolium, Brassica oleracea Var. Capitata, Cyperus rotundus, Plectranthus barbatus, Porophyllum ruderale (Jacq.) Cass e Sonchus oleraceus. Revista Eletrônica de Farmácia. 2008;5(1):101-110, doi: 10.5216/ref.v5i1.4620.

11. Pastore JFB, Mota MCA. Rhaphiodon In Flora do Brasil 2020 em construção. Jardim Botânico do Rio de Janeiro [internet]. 2020 mai [acesso em 13 mai 2020]. Disponível em: http://www.floradobrasil.jbrj.gov.br/reflora/floradobrasil/FB8295

12. Pio IDSL, Lavor AL, Damasceno CMD, Menezes PMN, Silva FS, Maia GLA. Traditional knowledge and uses of medicinal plants by the in habitants of the islands of the São Francisco river, Brazil and preliminary analysis of Rhaphiodon echinus (Lamiaceae). Braz J Biol. 2019 Jan;79(1):87-99, doi: 10.1590/1519-6984.177447.

13. Mansur JS, Breder MNR, Mansur MCA, Azulay RD. Determinação do fator de proteção solar por espectrofotometria. An Bras Dermatol. 1986;61(3):121-124.

14. Sayre RM, Agin PP, LeVee GJ, Marlowe E. A Comparison of in vivo and in vitro testing of sunscreening formulas. Photochem Photobiol. 1979 Mar;29(3):559-566, doi: 10.1111/j.1751-1097.1979.tb07090.x.

15. Brasil. Ministério da Saúde, Agência Nacional de Vigilância Sanitária. Resolução RDC $\mathrm{N}^{\circ} 30$, de $1^{\circ}$ de junho de 2012. Aprova Regulamento Técnico do MERCOSUL sobre protetores solares em cosméticos. Diário Oficial da União. Brasília. 4 jun 2012;(Seção 1):83-84.

16. Medeiros MAC, Simões MM, Santos B, Marques FMC, Leite MFMS, Oliveira HMBF, et al. Avaliação da atividade fotoprotetora do extrato etanólico de Rhaphiodon echinus Schauer. Res Soc Develop. 2020 Mai;9(7):e585974410, doi: 10.33448/rsd-v9i7.4410.

17. Simão BLA, Simão KLA, Pereira CT, Alves MS, Medeiros MAA, Souza MSAS, et al. Avaliação da atividade fotoprotetora e antioxidante do extrato etanólico de Praxelis clematidea (GRISEB.) R. M. King \& H. Robinson (Asteraceae). Int J Develop Res. 2019 Out;9(10):30291-30294.

18. Campelo PMS. Plantas medicinais e seus extratos: a necessidade de estudos continuados. Estudos Biol. 2004 Jan/Mar;26(56):1-2, doi: 10.7213/reb.v26i56.21739. 